\title{
Physiological assessment of pilot workload in simulated and actual flight environments
}

\author{
ERNEST LINDHOLM and NORWOOD SISSON \\ Arizona State University, Tempe, Arizona
}

\begin{abstract}
Recent advances in microcomputer technology make it possible to monitor the internal state of the human operator in exotic environments with an ease and accuracy not imagined 10 years ago. It is now economically and technically feasible for many psychophysiologists to move out of the laboratory and apply their expertise to real-world problems. Two applications of psychophysiological methods to the study of pilot workload are described, one in simulated flight and one in actual flight.
\end{abstract}

Traditionally, the study of operator workload has fallen within the research domain of the experimental psychologist, and in the years following World War II, dual-task methodology became the most popular approach. A problem with the dual-task method is that it is intrusive; that is, the secondary task intrudes upon the operator's ability to perform the primary task, and therefore it is often not clear whether performance decrements are due to central processing limitations, response interference, or both. Another problem is that intrusive techniques cannot be ethically applied in situations in which interference with primary task performance might compromise the safety of the operator. For these reasons, psychophysiological methods appear to many to be a preferable alternative to the dual-task approach. The psychophysiological method can be viewed as nonintrusive, since the operator is not required to perform any task that is out of the ordinary and workload is assessed in terms of metabolic response, rather than performance decrements. This permits workload evaluations in real-world situations, such as piloting a high-performance aircraft.

Highly realistic computer simulations of ships, ground vehicles, and aircraft are widely used so that the human operator can experience, and receive training in, situations far too dangerous to practice in reality. Thus, technology must be developed to assess workload in both simulators and the actual machines being simulated. Two applications are now described, one in an aircraft simulator and one in an actual aircraft.

\section{APPLICATION IN A SIMULATOR}

\section{Introduction}

The Advanced Simulator for Pilot Training (ASPT), lo-

This research was supported by Air Force Contracts F33615-80-C0020 and F33615-81-C-0005 and by research funds provided by Arizona State University. The authors' mailing address is: Department of Psychology, Arizona State University, Tempe, AZ 85287. cated at Williams Air Force Base near Chandler, Arizona, is a highly realistic, full-cockpit simulation of the F-16 aircraft. Computer-generated images displayed on large cathode-ray tubes provide complete visual simulation of mountains, buildings, trees, and other environmental features. Any environment can be displayed and any mission can be programmed by means of software changes. Since the ASPT is heavily used by many different researchers, it is not possible for any one researcher to install recording devices, amplifiers, or other hardware in the cockpit. Therefore, in this environment, the decision was made to use a combination of off-the-shelf biotelemetry devices and custom-fabricated logic devices to record event-related potentials (ERPs), eye blinks, heart rate (measured as interbeat intervals, or IBIs) and respiration rate while pilots flew a simulated mission through a hostile threat environment.

ERPs were derived from the vertex referenced to right mastoid and with left mastoid as ground. Eye movements were derived from the superior orbital ridge and the canthus of the left eye. Heart activity was derived from leads placed on the sternum and left lateral rib. Beckman silver/silver-chloride electrodes and Beckman doubleadhesive collars were used for all placements except the vertex, which utilized the Beckman electrode in conjunction with Grass electrode cream and a gauze pad. Respiration activity was derived by placing a thermistor in a simple dust mask of the type found in any hardware store. This covers the mouth and nose, and the thermistor is warmed on exhalation and cooled on inspiration. This method is preferred to the chest strain gauge technique, since the latter is subject to many different types of movement artifacts (torso twisting, lifting the arms, leaning to right or left, etc.).

The hostile threat environment required the pilot to make a low-altitude flight into enemy territory with the goal of bombing a small rectangular building. The building was protected by four surface-to-air missile (SAM) sites. Every $3 \mathrm{sec}$, the pilot heard a 250 -msec tone over 
the headset; these tones gave the pilot critical information regarding the level of SAM threat. A $1000-\mathrm{Hz}$ tone signaled "safe" (no SAM threat), a 1250-Hz tone signaled "acquisition" (SAM radar has detected the aircraft), a 1500-Hz tone signaled "track" (the SAM radar has locked onto the aircraft), and a $1750-\mathrm{Hz}$ tone signaled "launch"' (a SAM has been launched against the aircraft).

Thirty-four rated Air Force pilots flew 10 missions each. Each mission lasted approximately $3 \mathrm{~min}$, and there was a 1-min break between missions. The goal was to bomb the target without being hit by a SAM.

\section{Hardware}

The multichannel telemetry device (UFI Corp., Morro Bay, CA) consisted of three component groups: (1) a miniature battery-powered signal conditioner, modulator, and transmitter weighing about $1 \mathrm{lb}$ and carried on the pilot's person, (2) a receiver and demodulator, and (3) a four-channel AM tape deck. The receiver, demodulator, and tape deck were located in a roll-away cabinet that, during data-collection sessions, was positioned in the ASPT computer console room about $50 \mathrm{ft}$ from the pilot. The roll-away also contained an oscilloscope for monitoring the integrity of the biological signals, an audio oscillator capable of generating the four threat tone frequencies, and a microcomputer. The microcomputer was custom fabricated by the second author, but nearly any low-cost, off-the-shelf unit could perform the same functions of simple switching and decision making. The microcomputer accepted, as input, logic signals from the large ASPT mainframe indicating which of the four threat levels was currently active. One output of the microcomputer then triggered the appropriate oscillator to deliver the tone burst to the pilot. In this manner, only two physical cables were associated with the roll-away, one cable that conveyed logic pulses from ASPT to the roll-away and one audio cable that was strung from the roll-away to the pilot's headset in the cockpit. Both cables were disconnected and stored in the roll-away, and the roll-away itself was stored in an unused corner of the room between data-collection sessions.

The biotelemetry transmitter uses pulse-width modulation to code the biological signals. Channel 1 of the fourchannel AM tape deck was modified to allow the recording of inputs with fast rise times. The demodulator was not used; instead, the pulse-width-modulated signal was recorded directly on Channel 1 . Thus, all four biological signals were recorded, in modulated form, on just one channel of the AM tape deck. This method circumvents the great expense involved in the purchase (and maintenance) of an FM instrumentation tape system.

Channel 2 of the tape deck was used for event coding (freeze, release from freeze, bomb release). These events were simply relayed by the microcomputer from the ASPT mainframe to the tape deck inputs. The microcomputer also sent a binary code to Channel 3 of the tape deck to indicate which tone (and therefore which threat level) was currently active. Channel 4 of the tape deck was used for voice commentary.

A duplicate tape deck, receiver, and demodulator were located in the authors' laboratory at Arizona State University (ASU). Only the reel of tape had to be transported between sites. The ASU laboratory is equipped with a PDP-11/34, A/D converters, and hard disks. Reading the data from AM tape amounted to routing Channel 1 of the tape through the demodulator and then into four A/D inputs of the PDP-11/34. Channels 2 and 3 were simultaneously fed to a serial line interface. Author-written software read the $A / D$ channels and the serial port and organized all data into files suitable for later analysis.

\section{Results}

The system described proved remarkably free of hardware problems, and the physiological data were as free of electrical noise as any recorded in the authors' laboratory using an electrically shielded booth. Detailed results will be reported in a later publication, but some preliminary results can be summarized here.

The threat tones were of short duration and occurred every $3 \mathrm{sec}$; thus, they served as ideal time markers to analyze physiological change throughout the mission. A premise of the experiment was that increased threat, although simulated, would increase workload because the pilot would be required to process more information when threat was high than when threat was low. That is, the pilots were already in a high cognitive workload situation; they had to maintain high speed at low altitude and prepare for target acquisition and weapon delivery. Increased SAM threat added to this workload, since the pilot had to plot a mental strategy to reduce the level of SAM threat (e.g., "Should I reduce altitude further and risk a crash? How long has that track tone been on? Is a launch imminent?").

The ASPT is a highly visual environment involving considerable head and eye movement by the pilot. Not surprisingly, vertex event-related potentials (ERPs) evoked by the threat tones were severely contaminated by eye movements, and nearly half of the data could not be analyzed. Given this limitation, the results indicated that threat level affected both the N2 and P3 components of the ERP: N2 amplitude became more negative, P3 amplitude became more positive, and P3 latency increased as a function of increasing threat level. All three functions were monotonic and statistically significant. The heart and respiration signals were less affected by movement artifact. Heart rate and respiration rate increased monotonically and significantly as a function of increased threat level.

The results indicate that it is practical to apply psychophysiological techniques in simulated operational environments. Autonomic nervous system variables (heart, respiration) are little affected by movement artifact, but 
the ERP is greatly affected by movement artifact. One might expect as much as 50\% ERP data loss in a highly active environment such as the ASPT.

\section{APPLICATION IN AN OPERATIONAL A-7 AIRCRAFT}

\section{Introduction}

In this application, which is now entering the datacollection phase, a dual-seat version of an A-7 aircraft is slightly modified to permit in-flight recording of heart rate and respiration rate, plus 23 aircraft positional and performance variables. The performance site is the Fighter Weapons School of the Arizona Air National Guard, located in Tucson, about 100 miles from the authors' laboratory. Only one pilot (Maj. Milt Miller) is being studied at present. An extremely important consideration is the cooperation of the pilot, and in this case, Major Miller is not just cooperative, he also leads the project. His expertise is in low-level, high-velocity flight, and he trains other pilots in these techniques. Without question, these are high-workload flight segments, and unlike in simulation, there are very real threats to life and property. For example, Major Miller frequently reaches speeds in excess of 450 knots at altitudes of $50 \mathrm{ft}$ or less.

\section{Hardware}

In this application, all hardware is custom designed and fabricated by Interface Research Associates, Scottsdale, Arizona. No off-the-shelf devices are available that can meet the technical needs of the application and also meet the physical space limitations specified by Air Guard personnel for the A-7 aircraft.

The current system consists of two hardware units, a miniaturized physiological data-acquisition device (called the "pilot box") and a large unit that resides in the avionics equipment bay, called the "silver box" (so named because it is contained in an aluminum case). The pilot box is designed around a Hitachi HD 68P05V07 microprocessor. Physiological data are A/D converted at this stage to minimize the noise problems. The pilot box outputs 2400 -baud asynchronous phase-encoded physiological data to the silver box through a hard cable that runs from the cockpit to the avionics bay. The silver box is organized around a Z80 processor with $1 \mathrm{~K}$ RAM and 4K EPROM. This unit receives the phase-encoded data from the pilot box and also reads NRZ signals from the A-7 flight computer at $50 \mathrm{~K}$ bits $/ \mathrm{sec}$ and outputs 4800 -baud asynchronous phase-encoded data to one audio track of the heads-up-display (HUD) video cassette recorder (VCR). The 4800 -baud limit is set, not by our electronics, but by the bandpass of the HUD VCR. Much higher baud rates (and therefore the recording of more variables) could be realized by replacing the HUD VCR with a recorder of higher technology.

Although the pilot box can be carried in the flight suit, in this application it is secured in the map case situated by the pilot's right thigh. Its power is derived from the aircraft $28 \mathrm{~V} \mathrm{dc}$, which is reduced to $+12 \mathrm{~V} \mathrm{dc}$ and $-12 \mathrm{~V} \mathrm{dc}$ for the analog circuitry and $+5 \mathrm{~V} \mathrm{dc}$ for the digital circuitry. Heart activity is led from three disposable pregelled electrodes placed in a triangular pattern around the pilot's left breast. Respiration activity is detected by a thermistor placed in the pilot's air-hose connector. The thermistor is in series with a resistor, and a dc voltage is across the pair. The resulting current warms the thermistor, and inhaling draws cool air across the thermistor, reducing its temperature. This changes the thermistor's resistance, and the voltage oscillation at the node between the thermistor and resistor is sampled as the representation of respiration activity.

Both the heart and respiration activity are amplified and low-pass filtered, and then multiplexed and digitized. The Hitachi microcomputer averages the digitized signals and transmits 100 heart and 10 respiration values per second over a Manchester phase-encoded 2400-baud asynchronous data link with transformer isolation to the silver box. The latter is software programmable to select any subset of 23 variables from the 100 variables available from the A-7 flight computer and reduces the data rate from $25 \mathrm{samples} / \mathrm{sec}$ to $5 \mathrm{samples} / \mathrm{sec}$. Thus, aircraft variables (e.g., airspeed, altitude, $x-y$ coordinates, etc.) are sampled every fifth of a second. The final function of the silver box is to multiplex the biological data and the aircraft data. The multiplexed signal is recorded on one of the two audio channels of the HUD Umatic format VCR.

Following a data-collection period, the cassette tape is returned to the authors' laboratory, which contains, in addition to the PDP system described above, a tape playback unit and TV monitor. As the tape is played, the flight, as seen by the HUD TV camera, appears on the monitor. The pilot's voice comments are heard on audio Channel 1, and the data are decoded into the computer from audio Channel 2. In this manner, the entire flight can be reconstructed.

\section{Results}

As indicated above, the data-collection phase is just beginning. The hardware has been checked in the aircraft and functions well, although it was necessary to add 400$\mathrm{Hz}$ filters to the silver box circuitry in order to reduce electrical noise generated by the aircraft.

A major advantage of the system is its flexibility: A software change in the silver box can alter the aircraft variables studied, and relatively minor hardware changes in the pilot box can alter the physiological variables studied. Also, very little modification of the aircraft is required (an important consideration, since much red tape is involved in modifying military aircraft for research purposes): One cable was installed to connect the pilot box to the silver box, one slot was freed up in the avionics bay to accommodate the silver box, one $28-\mathrm{V}$ dc outlet was installed next to the pilot's seat to power the pilot box, and one short cable was installed from the silver box to the HUD VCR. 


\section{GENERAL CONSIDERATIONS}

Hardware costs (excluding the PDP computer system) for either of the above described applications are in the range of $\$ 15,000$ to $\$ 20,000$. However, these are first-time costs, and modifications of the hardware for other experiments and other applications would be only a fraction of this amount. Nonlaboratory research is always less convenient than laboratory research because of the travel involved and the fact that datacollection sessions are scheduled at the convenience of the pilots, not the experimenters. Nonetheless, workload assessment in field environments is an important and relatively untouched research area that needs and welcomes the special talents of the psychophysiologist. 